\title{
Granulomatosis with polyangiitis a challenge for diagnosis and treatment - case report
}

\author{
Anca Codreanu ${ }^{1}$, Madalina Ionela Chiriac ${ }^{2}$, Elena Rezus ${ }^{*, 1,2}$ \\ ${ }^{1}$ Rheumatology Department, Rehabilitation Hospital lasi, 2 "Grigore T. Popa" University of Medicine \\ and Pharmacy, lasi, Romania
}

\begin{abstract}
In this article we report the case of a 46 year-old male presenting with pulmonary, sinusal, otic, nasal and laryngeal lesions. He was diagnosed in December 2013 with granulomatosis with polyangiitis and we tried to achieve remission with cyclophosphamide and glucocorticoids. The onset of the disease included pansinusitis accompanied with mastoiditis and paralysis of the left facial nerve, bilateral hearing loss and episodes of intense headache. The patient underwent two surgeries to solve the Ear-Nose-Throat (ENT) area symptoms which have delayed the diagnosis and the initiation of appropriate therapy.
\end{abstract}

Keywords: granulomatosis with polyangiitis, ENT, necrotizing glomerulonephritis, "saddle" nose.

\section{Introduction}

Granulomatosis with polyangiitis or Wegener's granulomatosis (WG) is a systemic vasculitis with necrotizing small vessels, eosinophilic granulomatosis and microscopic polyangiitis (Churg-Strauss syndrome) [1]. The disease affects the arterioles, the capillaries or the venules, especially of the upper and lower respiratory tract and of the kidneys, leading to ischemic or hemorrhagic manifestations [1, 2].

Ninety two percent of patients have impairment of upper respiratory system or inner ear [2] represented by: rhinorrhea, nasal crusting, nasal pyramid collapse ("saddle" nose), pansinusitis, inner or media otitis, together with sensorineural deafness, paralysis, chronic mastoiditis with secondary facial nerve paralysis caused by the bone destruction.

Received: August 2014; Accepted after review: September 2014; Published: September 2014

*Corresponding author: Elena Rezus, Rheumatology Department, Rehabilitation Hospital, lasi, "Grigore T. Popa" University of Medicine and Pharmacy, lasi, Romania. Email: elena rezus@yahoo.com
In the absence of accurate treatment, the vasculitis progresses fast, mainly including the lower respiratory tract and the kidney structures, leading to a high rate of morbidity and mortality.

\section{Case report}

We present the case of a 46 year-old male who presented in the Rheumatology Department of Rehabilitation Hospital lasi in early 2014 after long medical investigations, especially in the ENT service. The patient was initially diagnosed with chronic hypertrophic rhinitis and pansinusitis.

The symptoms began in June 2013 with left hemicrania, rhinorrhea, nasal congestion and sero-mucinous secretions which determined the patient to self-administration of analgesics and NSAIDs.

In September 2013, a facial computerized tomography (CT) examination was performed revealing frontal and maxillary sinusitis and left mastoiditis. The patient is directed to the ENT Surgery Department of Bucharest Hospital where an anterior left ethmoidectomy was 
performed, with minimal improvement of the symptoms after surgery.

In November 2013, the nasal occlusive syndrome got worse associating muco-purulent rhinorrhea and left purulent otorrhea, sleep disorders, headache and paresis of the left facial nerve. The biological tests showed that the patient had an important inflammatory syndrome $(E S R=105 \mathrm{~mm} / \mathrm{h}, \mathrm{CRP}=24.37$ $\mathrm{mg} / \mathrm{dl})$.

In the ENT Department of Targu Mures Hospital, a new cranio-cerebral CT examination revealed mucosal thickening of the left and right maxillary sinus, almost completely obstructed of ethmoid and left mastoid cells by thickening mucosa and inflammatory exudate, inflammation in the middle ear without affecting the left ossicular chain (Figure 1 and Figure 2).

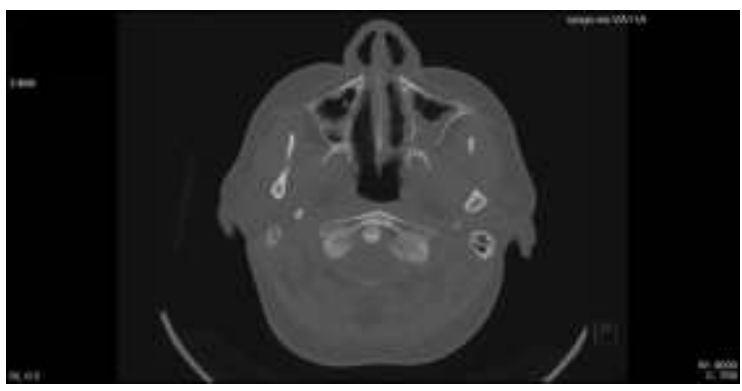

Fig. 1. Maxillary sinusitis in tranversal section of craniocerebral - CT.

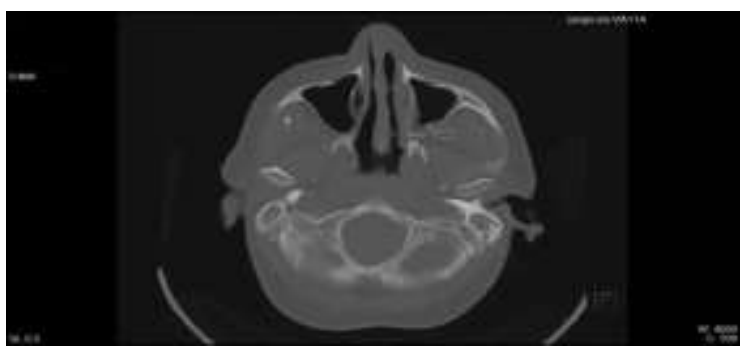

Fig. 2. Inflammation in the middle ear without affecting left ossicular chain in tranversal section of craniocerebral - CT.

After several days of antibiotics, surgery was performed and mucosal fragments were biopsied for histopathological examination.

The biopsy of the sinus mucosa (maxillary, ethmoid, frontal, sphenoid) showed multiple areas of ulceration with moderate edema and mixed inflammatory infiltrate, extensive vasculitis phenomena with the presence of fibrinoid necrosis and strong intramural inflammatory infiltration with neutrophils and formation of microabscesses; some vascular lesions leading to the appearance of geographic necrosis; "palisades" of histiocytes and multinucleated giant cells forming granulomas, imprecisely delineated.

The pathologist conclusion was that the description is suggestive for granulomatosis with polyangiitis (Wegener's granulomatosis). The patient was directed to a rheumatologist who initiated cortisone treatment (prednisone $50 \mathrm{mg} /$ day) and gastric protection drugs.

The clinical examination at the first hospitalization in lasi (January 2013) revealed bilateral hearing loss predominantly on the left side, left hemicrania, cough, dizziness, and purulent rhinorrhea. It also revealed the deformation of the nasal pyramid with "saddle nose" (Figure 3).

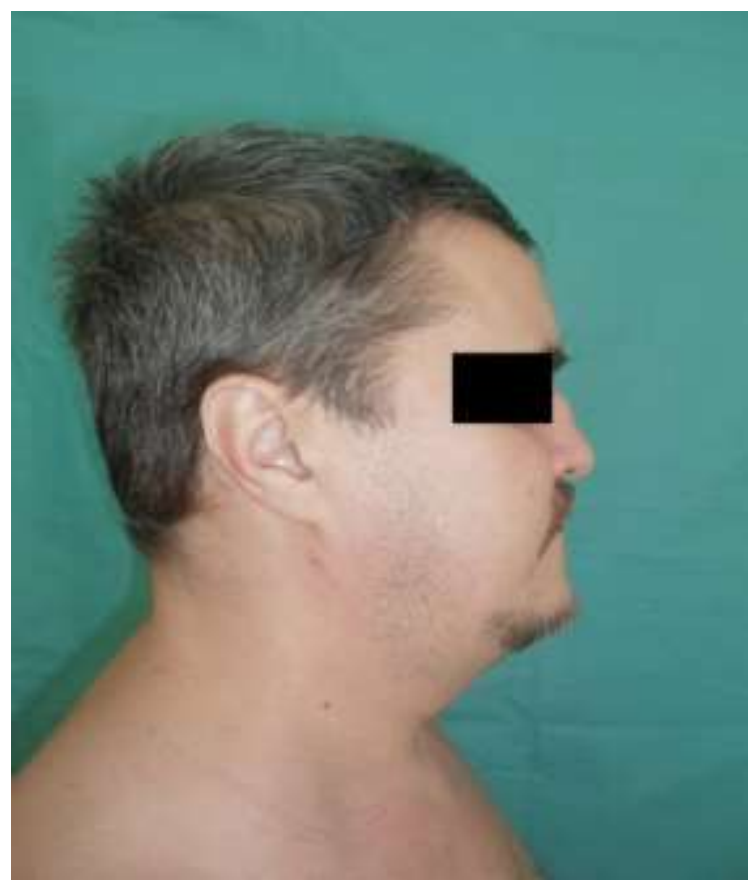

Fig. 3. "Saddle" nose profile

The biological examinations revealed moderate leukocytosis $\left(\mathrm{WBC}=15050 / \mathrm{mm}^{3}\right)$, inflammatory syndrome $\quad(E S R=30 \mathrm{~mm} / \mathrm{h}$, $\mathrm{CRP}=0.40 \mathrm{mg} / \mathrm{dl}$ ), mild hypercholesterolemia (cholesterol=212 $\mathrm{mg} / \mathrm{dl}$ ). Specific antibodies for Wegener's granulomatosis (c-ANCA and pANCA) were in normal limits. ENT examination described exacerbation of chronic left pansinusitis, chronic hypertrophic rhinitis, left 
facial nerve paralysis caused by left otomastoiditis and right chronic sero-mucinous otitis.

The recommendations included transtympanic bilateral aerators, left mastoidectomy for facial nerve decompression, saline nasal toilet and antibiotherapy (fluoroquinolons) for 7 days. Audiometry was performed and revealed bilateral neurosensorial hearing loss, predominantly of the left ear. In order to evaluate the lung function, a chest x-ray was performed which turned out normal.

The differential diagnosis included small vessels vasculitis (eosinophilic granulomatosis and microscopic poliangiitis). Also, respiratory and sinus infections, lymphoproliferative or neoplasic processes were excluded (craniofacial CT examination, histopathological examination, and ENT repeated examinations).

The septum perforation with subsequent collapse of the nasal pyramid ischemia caused by vasculitis led to differential diagnosis of cocaine exposure (detailed case history).

\section{Diagnosis}

At the moment, the case fulfills the American College of Rheumatology -1990 (ACR-1990) criteria for the diagnosis of granulomatosis with poliangiitis - WG (severe ENT damage: polysinusitis, muco-purulent chronic rhinitis, serous otitis, left mastoiditis with paralysis of the facial nerve, collapse of the nasal pyramid "saddle nose", all confirmed by histophatologycal examination). Also, the biological tests revealed leukocytosis and inflammatory syndrome, with normal parameters of immunological markers (cANCA, pANCA).

In order to assess the disease activity score we used the Birmingham Vasculitis Activiy Wegener's Granulomatosis for Modified Score (BVA/WG) that comprises 34 items gathered in nine systems. During the first period of hospitalization, the patient had a generalized and severe form of illness according to the European Study Vasculitis (EUVAS) classification (sinus damage, neurosensorial hearing loss, facial nerve palsy and nasal crusts).

\section{Treatment}

After the diagnosis of systemic involvement, early remission was attempted through the initiation of immunosuppressive treatment with cyclophosphamide pulse-therapy and glucocorticoids in doses of $1 \mathrm{mg} / \mathrm{kg} /$ day. We also prescribed trimethoprim-sulfamethoxazole, $480 \mathrm{mg}$ every 12 hours for 3 consecutive days, for Pneumocystis carinii (Pneumocystis jiroveci) infection's prophylaxy. The patient was discharged with further indication of corticosteroid treatment (methylprednisolone 64 $\mathrm{mg} /$ day).

A month later (February 2014), the patient had a good general condition, persistent bilateral hearing loss and predominantly left occurrence of mild myopathy in the thighs and arms. Biological tests revealed: leukocytosis $\left(\mathrm{WBC}=12710 / \mathrm{mm}^{3}\right)$, inflammatory syndrome (ESR=21 mm/h , CRP=0mg/dl) , mild liver injury $(A S T=49 \mathrm{U} / \mathrm{L}, A L T=28.3 \mathrm{U} / \mathrm{L}, \mathrm{GGT}=94.5$ $\mathrm{U} / \mathrm{L}$, negative $\mathrm{HBs} \mathrm{Ag}$, negative $\mathrm{HCV} \mathrm{Abs}$ ), modified muscle enzymes $(\mathrm{CK}=114.1 \mathrm{U} / \mathrm{L}$, $\mathrm{LDH}=297.4 \mathrm{U} / \mathrm{L}$ ), hyperglycemia (glucose $=127.2 \mathrm{mg} / \mathrm{dl}$ ) and mixed dyslipidemia (cholesterol=264.5 mg/dL, triglyceride $=281.63$ $\mathrm{m} / \mathrm{dl}$ ).

Re-examination of ENT showed persistent pansinusitis and left otomastoiditis with secondary facial nerve palsy, but with the improvement of bilateral hearing loss. Therefore, we maintain the recommendation for surgical treatment of the left otomastoiditis. We administered the second pulse-therapy (Cyclophosphamide $1000 \mathrm{mg}$ combined with glucocorticoids) and recommended the cortisone therapy after discharge (methylprednisolone $64 \mathrm{mg} /$ day slowly decreased to $48 \mathrm{mg} /$ dose/day).

In March 2014, the patient returned in our department for another cyclophosphamide pulse-therapy and the physical examination showed a more severe myopathy and muscular hypotrophy of the thighs and arms. Biological tests revealed: persistence of inflammatory syndrome and liver injury (AST=77 U/L, GGT= $326.9 \mathrm{U} / \mathrm{L}$ ), hyperglycemia (glucose $=142.3$ $\mathrm{mg} / \mathrm{dl})$, the emphasis of mixed dyslipidemia (cholesterol=277.6 $\mathrm{mg} / \mathrm{dL}$, triglyceride $=375.6$ $\mathrm{mg} / \mathrm{dl}$ ), modified muscle enzymes ( $\mathrm{CK}=28.7$ $\mathrm{U} / \mathrm{L}, \quad \mathrm{LDH}=409 \mathrm{U} / \mathrm{L})$, renal function and 
urinalysis tests were within normal limits. Reevaluation in otolaryngology service showed recurrent crusts of nasal mucosa.

In the context of the significant myopathy, a neurological examination was performed and the doctor recommended a Magnetic Resonance (MR) angiography of the brain and an electromyography.

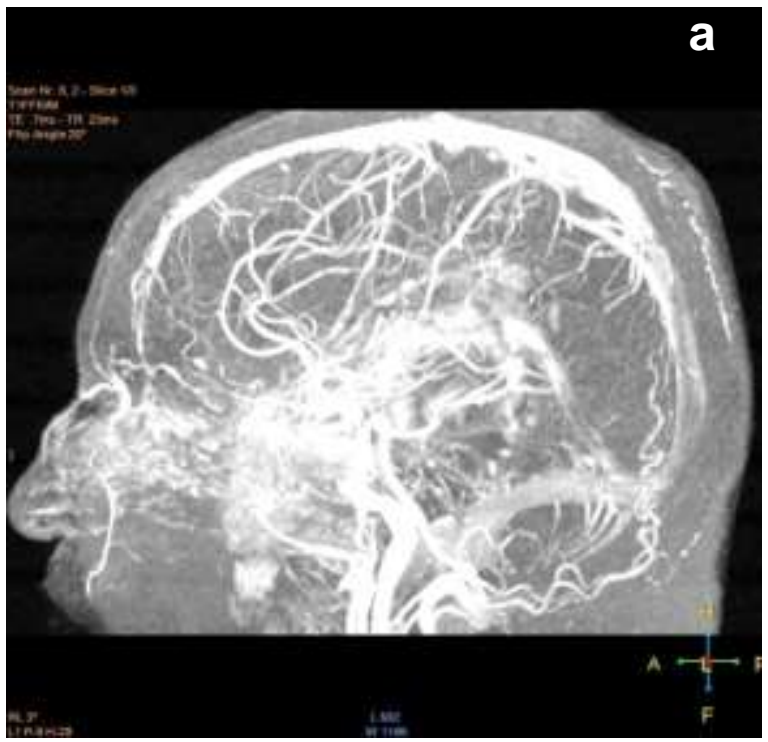

MR angiography revealed persistence of pansinusitis (maxillary, sphenoid and ethmoid sinuses), hypoplastic frontal sinus and left mastoid cells with hyperdens material, incompletely obstructed and constitutional changes of blood vessels (Figure 4.)

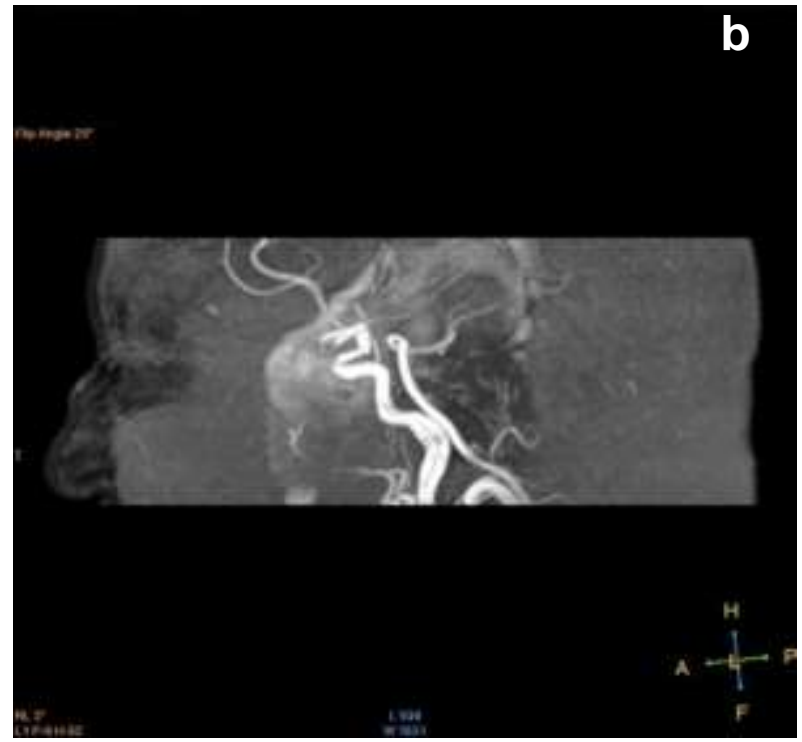

Fig. 4. MR angiography of the brain showed: a) hypoplastic frontal sinus and left mastoid cells incomplete obstructed by a hyperdens material; b) only constitutional changes of blood vessels

The electromyographic examination revealed myogenic changes (low amplitude potential, reduced intervals, myogenic interferential pathway) in the right vastus lateralis muscle, right anterior tibiae muscle, left gastrocnemius muscle, right deltoid muscle and in the left biceps muscle.

During hospitalization, the patient presented dry cough, moderate dyspnea, intense headache and fever over $38^{\circ} \mathrm{C}$.

Chest radiography was performed and described bilateral hilio-basal pulmonary infiltrates. For more detailed assessment, a chest-CT examination was performed, showing the appearance of "ground glass" opacity, mainly peripheral and perihilar in pulmonary parenchyma which appear "stained", some nodular densification with irregular outline both in base and periphery pulmonary parenchyma, aspect of interstitial lung injury caused by granulomatosis with polyangiitis (Figure 5 and Figure 6).

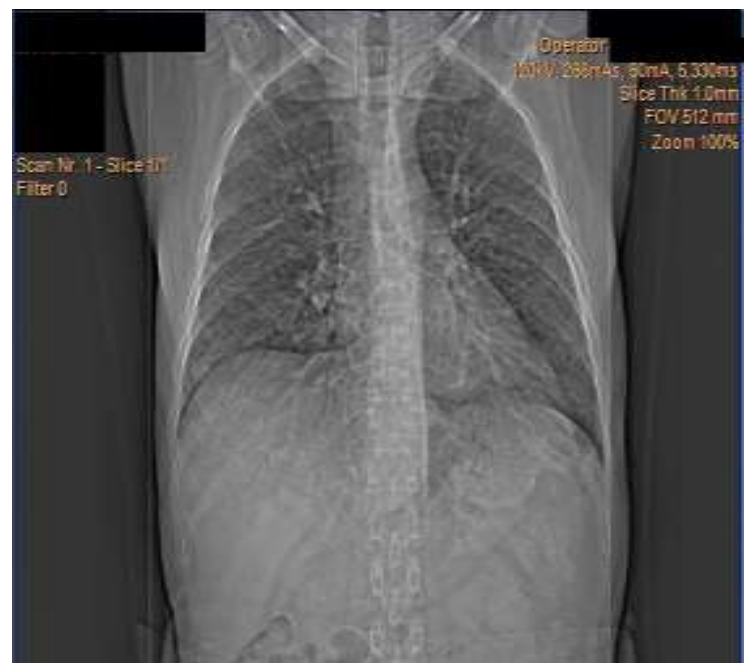

Fig. 5. CT scan (frontal section) reveals the appearance of "ground glass" opacity and pulmonary nodules. 


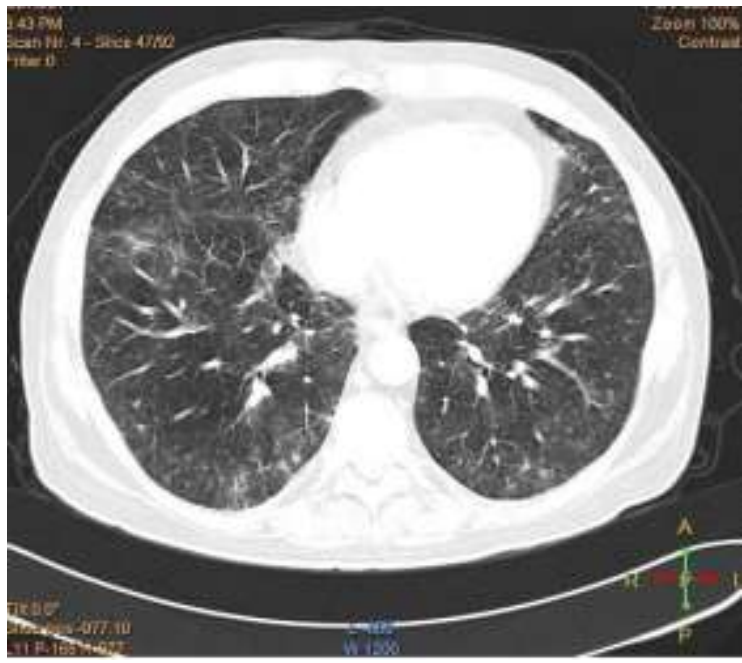

Fig. 6. CT scan (transversal section) reveals pulmonary nodules and aspect of interstitial lung injury caused by vasculitis

The re-examination of the current disease activity using the score BVA / WG revealed that the patient had four persistent symptoms (affected sinus, neurosensorial hearing loss, paralysis of the facial nerve, crusts of the nose) and two new symptoms (fever over $38^{\circ} \mathrm{C}$ and lung injury). Considering the pulmonary lesions, we consecutively administered three pulses of solumedrol $500 \mathrm{mg} /$ day after which, the febrile syndrome and other associated symptoms (cough, dyspnea) were remitted. $\mathrm{He}$ also received a third pulse of cyclophosphamide $1000 \mathrm{mg}$ (in association with corticosteroids) with favorable clinical and biological outcome.

\section{Discussion}

The first evidence of granulomatosis with polyangiitis was noted by Wegener in 1936 [1] who discovered a lesion of the vascular wall induced by inflammation which could lead either to thickening and secondary lumen stenosis with subsequent ischemia or to thinning of the wall and formation of aneurysms that can cause bleedings.

In $92 \%$ of the patients, impairments of the upper respiratory and ENT were observed [2]. The most common symptoms are: nasal crusts, rhinorrhea, erythematous nasal mucosa and deformation of the nasal pyramid caused by Kiesselbach plexus ischemia of the anterior septum. The cause for hearing loss of the middle ear is secondary to Eustachian tube dysfunction (nasopharyngeal injury). The chronic mastoiditis with facial nerve paralysis is usually associated to bone destruction.

In $10-20 \%$ of patients, subglottic stenosis appears as an effect of the vulnerability to chronic inflammation and restorative process of subglottic tissue [3] or to overactive recovery processes that lead to chronic fibrosis and subsequent stenosis [4].

The chest-CT examination (usually without contrast agents in order to protect the kidney function) highlighted the lung involvement: nodules, cavities, endobronchial stenosis and alveolar hemorrhage. The classic renal manifestation involved in WG consists of necrotizing glomerulonephritis that occurs in $25-75 \%$ of cases. Serum creatinine test in the first month of the treatment has predictive value for the prognosis of the patients [5]. WG is further being associated with various ophthalmological, dermatological, musculoskeletal, neurological, cardiovascular or gastrointestinal disorders.

The diagnosis is based on clinical examination and sustained by specific histopathological examination. An important role for diagnosis is assigned to anti-neutrophyl cytoplasmic antibodies (ANCA), c-ANCA (granular cytoplasmic immunofluorescence) being the immunological marker for this disease. In $80-95 \%$ of cases, PR3 c-ANCA are positive, their presence being associated with higher mortality [6], higher rate of disease relapse [7], and sudden kidney failure in patients with glomerulonephritis [8]. The treatment aims to decrease morbidity and mortality, maintaining remission for a long period, limiting the toxicity of the drugs used and is made accordingly to the stage of disease.

The particularity of the case is represented by the fact that the patient underwent two surgeries to resolve the ENT symptoms, which delayed the diagnosis and the initiation of the therapy. The specific antibodies ( $p$-ANCA and c-ANCA) were negative at the first admission, which suggested a good prognosis and no renal injury.

The induction of remission treatment with cyclophosphamide pulse-therapy combined with steroids didn't have a favorable clinical 
outcome. Therefore, we consider the patient to be a possible candidate for treatment with biological agents such as rituximab. Several clinical studies approved this biological therapy such as the RAVE study (experimental clinical trial, randomized, double-blind, double-coded, placebo-controlled, non-inferiority, the GPA or MPA patients received rituximab or standard treatment for 6 months in order to remission induction) or RITUXVAS (multicenter, openlabel, 2 groups, parallel design comprising newly diagnosed $\mathrm{AAV}$ and renal involvement).

\section{Conclusions}

The patient we are reporting was classified as suffering of an early systemic form of WG

\section{References}

1. Muller $\mathrm{K}$, Lin $\mathrm{JH}$. Orbital granulomatosis with polyangiitis (Wegener granulomatosis): clinical and pathologic findings. Arch Path Lab Med 2014; 138(8):1110-1114.

2. Hoffman GS, Kerr GS, Leavitt RY, et al. Wegener granulomatosis: an analysis of 158 patients. Ann Intern Med 1992; 116:488-498.

3. Maronian NC, Azadeh $\mathrm{H}$, Waugh $\mathrm{P}$, Hillel A. Association of laryngopharyngeal reflux disease and subglottic stenosis. Ann Otol Rhinol Laryngol 2001; 110:606-612.

4. Diegelmann RF, Evans MC. Wound healing: an overview of acute, fibrotic and delayed healing. Front Biosci 2004; 9:283-289.

5. Weidner S, Geuss S, Hafezi-Rachti S, Wonka A,Rupprecht HD. ANCA-associated vasculitis with renal involvement: an outcome analysis. Nephrol Dial Transplant 2004; 19:1403-1411.

6. Hogan SL, Nachman PH, Wilkman AS, Jennette $\mathrm{JC}$, Falk RJ and the Glomerular Disease Collaborative Network. Prognostic markers in patients with antineutrophil cytoplasmic and we attempted induction of remission with cyclophosphamide therapy, goal which was not achieved. Cyclophosphamide in pulse therapy shows the same results as the ones proven for per os treatment, but has a lower toxicity due to lower dosage accumulation and a higher risk for relapses $[9,10]$.

We also consider that the prognosis is reserved due to the development of new manifestations in the remission induction period, and side effects of corticosteroid treatment which appeared shortly after (steroid diabetes, myopathy still possible in this context). Therefore, the patient can be a possible candidate for treatment with biological agents such as rituximab. Several clinical studies approved this therapy, such as the RAVE study or the RITUXVAS study.

autoantibodyassociated microscopic polyangiitis and glomerulonephritis. J Am Soc Nephrol 1996; 7:23-32.

7. Booth $A D$, Almond MK, Burns A, et al. Outcome of ANCA associated renal vasculitis: a 5-year retrospective study. Am J Kidney Dis 2003; 41:776-784.

8. Franssen CFM, Gans ROB, Arends B, et al. Differences between anti-myeloperoxidase- and anti-proteinase 3-associated renal disease. Kidney Int 1995; 47:193-199.

9. de Groot K, Harper L, Jayne DRW, et al. Pulse versus daily oral cyclophosphamide for induction of remission in antineutrophil cytoplasmic antibody-associated vasculitis: a randomized trial. Ann Intern Med 2009; 150:670-680.

10. Harper L, Morgan MD, Walsh M, et al. Pulse versus daily oral cyclophosphamide for induction of remission in ANCA-associated vasculitis: long-term follow-up. Ann Rheum Dis 2012; 71:955-960. 